iRASD Journal of Management
Volume 2, Number 2, 2020, Pages $79-83$
JRASD
https://journals.internationalrasd.org/index.php/jom

\title{
Using of Smart Contracts in Logistic Information Systems Services and the Ways to Review it
}

Dr. Khaldoun Besoul ${ }^{1}$, Dr. Ayman Nayef Al Halaybeh ${ }^{2}$, Prof. Safwan Al Salaimeh ${ }^{3}$

${ }^{1}$ Computer science Department, Faculty of Science and arts/Tanomah campus, King Khalid University, Abha, KSA, Email: kbesoul@kku.edu.sa

${ }^{2}$ Computer science Department, Faculty of Science and arts/Tanomah campus, King Khalid University, Abha, KSA, Email: Aalhalaybeh@kku.edu.sa

${ }^{3}$ Department of Software Engineering, Faculty Information Technology, Aqaba, University of Technology, Jordan, Email: safwan670@yahoo.com

\begin{tabular}{l}
\hline ARTICLE INFO \\
\hline Article History: \\
Received: $\quad$ July 22, 2020 \\
Revised: $\quad$ Noverober 28, 2020 \\
Accepted: $\quad$ December 31, 2020 \\
Available Online: \\
\hline Keywords: \\
Development \\
Activities \\
Contracts \\
Technical \\
Improvement \\
Application \\
Logistics
\end{tabular}
\begin{abstract}
There are many changes related to the development of the Internet and e-business, which significantly impact the logistics sector's service sectors. Electronic logistics is not a new segment or a new standard in the classification process for logistics companies. It leads to the development and improvement of a wide range of logistics services. Blockchain technologies can be used to improve and develop services, which are an integral part of this evolution. This research sheds light on the mechanism and application of these technologies in logistics services and the extent of their impact on logistical activities where some technical aspects of Blockchain are studied in detail, including smart contracts that use the Ethereum platform as an example.
\end{abstract}

(c) 2020 The Authors, Published by iRASD. This is an Open Access article under the Creative Common Attribution Non-Commercial 4.0 Corresponding Author's Email: safwan670@yahoo.com

\section{Introduction}

Information and communication technology represents a fundamental lever for modernizing the public sector. In front of an environment that knows continuous transformations and various constraints, the administration finds itself, with all its components, called to adapt to this context to make the project of change brought about by technological means in the horizon of approving an electronic administration. It may contribute to positive changes in the relationship between management and its customers. It should be noted that the transition from traditional management to electronic management is always linked to creating the appropriate conditions and climate, so the best scenario for achieving a proper implementation of the electronic management strategy and achieving the effects and expected results from it is not achieved in management tainted by structural imbalances, so it is more appropriate to fix these imbalances and use information as a mechanism for renewal And development, just as the use of electronic management should be based on rational spending by evoking the cost of these electronic programs in exchange for the impact and role they target. The modern world requires a comprehensive and integrated development in the field of public institutions and business. A prominent trend in the development of our society is the digitalization of the economy. IT technologies and the Internet environment are becoming integral elements of logistics organizations' functioning (Batiha, Al Salaimeh, \& Besoul, 2007). The current state of the development of logistics requires a high level of computerization and automation in managing flow processes to ensure a competitive level of logistics process costs and quality. One of the promising areas providing these conditions is blockchain technologies (Yao, Zhang, \& Zhou, 2006). 
One of the results of technological progress and globalization is inevitable aspects that direct each political system towards defining some administrative methods that guarantee to win the citizens' consent while providing some services of high quality and in the least possible time within the available legal, regulatory framework, which prompted most countries to move towards the idea of digitization. Administration and public institutions to raise the level of public service provision. Thus, this goal has a vital link in providing a digital environment that activates electronic management by transforming society from the traditional image to an information society without affecting the values and traditions of that society. Simultaneously, it may constitute a clear challenge to the authorities in achieving the universalization of electronic management on all levels (Sula, Grishin, Kitachine, \& Reva, 2009).

\subsection{The relevance}

The relevance of this topic is too high. The toolkit of blockchain technologies is developing rapidly; however, in the field of science and the legislative sphere, an information vacuum and a time barrier have formed regarding implementation in the real business sector. It is also worth noting that there is no clear understanding of where the framework for using blockchain technologies is located. Many organizations are already successfully implementing blockchainbased projects, including IBM, Microsoft, Walmart, Maersk, and many other large economic entities of different orientations. But most of the projects are at the beta stage (AL SALAIMEH, 2018).

Purpose of the article: by identifying and structuring the categorical and conceptual apparatus of blockchain technologies, identify tools applicable in the field of logistics and conduct a technical review of these technologies using smart contracts.

\subsection{The tasks}

The purpose of the research determined the setting of the following:

- To streamline and identify the categorical and conceptual apparatus of blockchain technologies;

- Systematize information relevant to the research topic on the use of blockchain technologies in logistics;

- To reflect all the necessary theoretical and practical aspects of smart contracts as an example of the use of blockchain technologies;

\subsection{The scientific novelty}

At the moment, there is no specific scientific description and the corresponding standardization of the process of functioning of the DRM system based on, or with elements, blockchain technologies, which determines the scientific novelty of the research.

\section{The role of Blockchain technologies in logistics}

Blockchain technologies in logistics can give the right impetus to the implementation of the current state tasks. Within the digital economy, Blockchain in logistics can help the world achieve transparency and security. The peculiarities of this technology, which perfectly cope with this task, lie in the general availability, the absence of the possibility of changes, the impossibility of forging data (Allen, 1995). In logistics, this technology allows you to track all processes from start to finish, for example, from the delivery of goods from manufacturer to consumer. In this case, all ongoing operations are recorded and added as a new element to the chain with the assignment of a unique number using complex mathematical algorithms. The new element stores all information, including information about the previous one, hence the name block - block and chain - chain. A blockchain is essentially a ledger of fact records replicated across multiple devices (computers) in a peer-to-peer network (P2P). One node is called a "node." Facts can be any digitized information units. Network members are anonymous individuals called nodes. Communication processes within this network are protected by cryptographic encryption to ensure reliable identification of the recipient and sender of information. Adding facts to the 
register requires reaching a consensus between the network nodes; this consensus is called a block (Al Halaybeh, Besoul, \& Al Salaimeh; Al Salaimeh, 2017).

In more detail, let's consider some of the technological and technical aspects of blockchain technologies, namely smart contracts. Let's take the Ethereum platform as an example since Ethereum is the most interesting in the B2B market. This interest is due to an optimized system for the functioning of smart contracts inside the Ethereum blockchain. Ethereum's blockchain platform is an individual system of transaction states. Ethereum's state machine has billions of different transactions, with all transactions grouped into blocks so that each block contains information about the previous block. Thus, a chain of blocks is formed (hence the name "blockchain") (Figure 1).

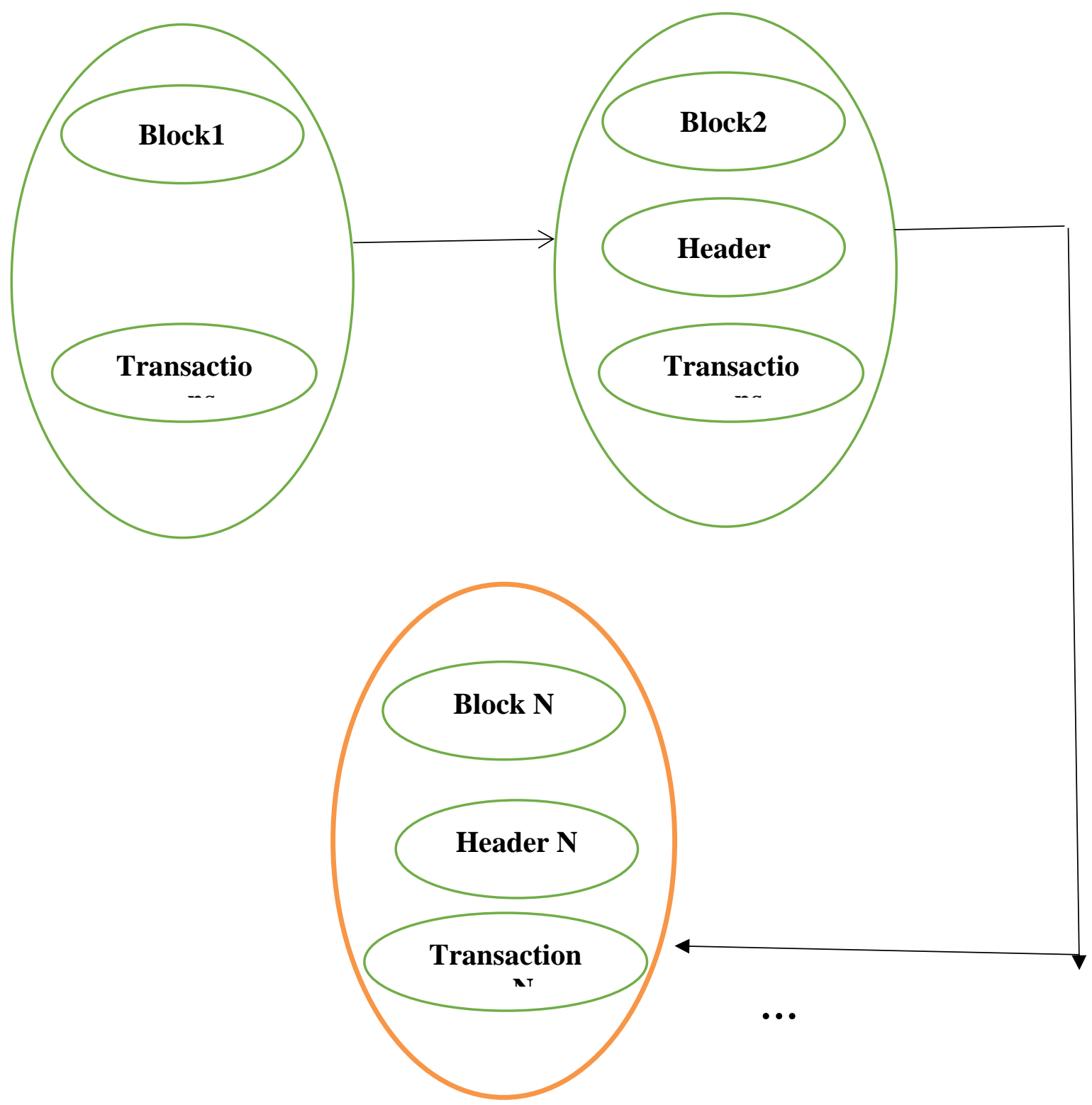

Figure 1: Ethereum blockchain

Essentially, Ethereum's state machine consists of 2 smaller building blocks, an external account and an account node, which interact with each other using a messaging model. Transactions from this perspective are moving the message (Al Salaimeh, 2017, 2018).

With an offshore account, you can send messages to both other offshore and contract accounts. A private key is required for this transaction. The contract account, in turn, is virtually self-contained. That is, it is possible to initiate certain transactions only in response to incoming transactions. It is not possible to initiate new transactions with a contract account. As mentioned above, smart contracts are one of the essential tools in the B2B marketplace, especially in finance and logistics. The content of smart contracts can refer to an algorithm that precisely 81 
follows mathematical reasoning. Smart contract management can only be implemented within the algorithm stipulated in the smart contract. For example, if you do not provide a function that self-destructs a smart contract when writing a smart contract, then that smart contract is conditionally perpetual. The reaction process for the node registers is shown in figure 2 .

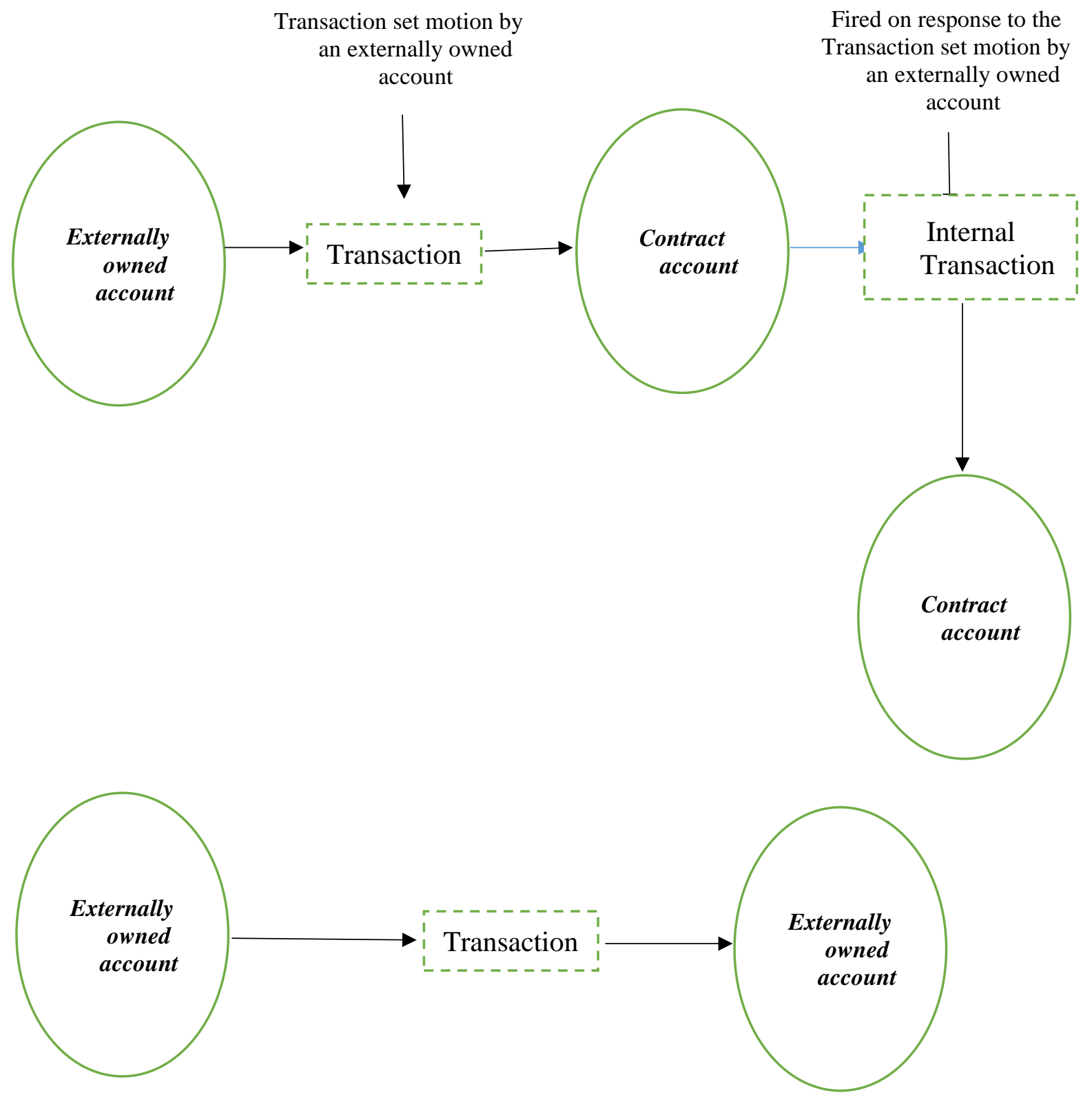

Figure 2: Relationship between contract accounts.

It should be noted here that the process of editing a smart contract itself is straightforward. However, editing the smart contract with no security holes for hackers' breaches and the lack of vulnerabilities in the smart contract system requires a thorough analysis of all possible algorithms that the smart contract contains. They can be useful tools in this case, such as network graph theory, matrix analysis, and other economic and mathematical methods. As mentioned above, after launching a node in the blockchain system, it only works within the limits of the algorithms embedded in it (Al Salaimeh, Al Saraireh, \& Al Rawashdeh, 2015).

At present, many informational sources offer smart contract models of a different nature and may contain vulnerabilities. Also, some standards are formed for writing smart contracts, compliance with which can guarantee reliability for counterparties (Al Salaimeh \& Batiha, 2006; Wang, Lu, \& Kvam, 2006). 


\section{The results}

Through the preceding, we see that this research has shed light on the mechanism and application of blockchain technologies in logistics services and the extent of their impact on logistical activities. Some of the technical aspects of Blockchain have been studied in detail, including smart contracts that use the Ethereum platform as an example.

\section{Recommendation conclusion}

From the above, we see that the blockchain system and its associated tools are primarily compatible with logistics services' objectives. Through the use of blockchain technologies, we believe that it is possible to reduce the costs of managing all logistical flows and improve the flow of operations.

Even more impressive for enterprises are smart contracts, which are best executed using the Ethereum platform. The presence of smart contracts in the Ethereum platform sets it apart from other popular blockchain platforms. It should be noted that logistics is one of the most promising industries to use blockchain technologies, particularly the Ethereum platform and smart contracts.

\section{References}

Al Halaybeh, A. N., Besoul, K., \& Al Salaimeh, S. Development of a Model for Monitoring and Analysis of Road Traffic Using an Algorithm for Neural Networks.

Al Salaimeh, S. (2017). The Optimization Problems of Informational Servicing Logistics Systems by Using Queuing Theory. International Journal of Scientific Research in Computer Science, Engineering and Information Technology, 2(6).

AL SALAIMEH, S. (2018). Mathematical description of resources distribution for logistics information systems. Leonardo Journal of Sciences(32), 1-9.

Al Salaimeh, S., Al Saraireh, Z., \& Al Rawashdeh, J. H. (2015). Design a model of language identification tool. International Journal of Information \& Computation Technology, 5(1), 11-18.

Al Salaimeh, S., \& Batiha, K. (2006). Business process simulation with algebra event regular expression. Information Technology Journal, 5(3), 583-589.

Allen, J. (1995). Natural language understanding: Pearson.

Batiha, K., Al Salaimeh, S., \& Besoul, K. (2007). Digital art and design.

Sula, A., Grishin, V., Kitachine, Y., \& Reva, M. (2009). Diagnostic method utilizing standard lead ECG signals. In: Google Patents.

Wang, N., Lu, J.-C., \& Kvam, P. (2006). Reliability modeling in spatially distributed logistics systems. IEEE Transactions on reliability, 55(3), 525-534.

Yao, D. D., Zhang, Q., \& Zhou, X. Y. (2006). A regime-switching model for European options. In Stochastic processes, optimization, and control theory: applications in financial engineering, queueing networks, and manufacturing systems (pp. 281-300): Springer. 